\title{
Linealización mediante Predistorsión Digital de un Sistema Radio sobre Fibra de Doble Banda
}

\author{
Carlos Mateo, Nieves Pérez, Paloma García, Pedro L. Carro, Jesús de Mingo, \\ Íñigo Salinas \\ Grupo de Comunicaciones Móviles (GCM) \\ Instituto de Investigación en Ingeniería de Aragón (I3A) \\ Universidad de Zaragoza, Mariano Esquillor s/n, 50018, Zaragoza, Spain. \\ Tel. +34-976762707, e-mail: cmperez@unizar.es
}

\section{Resumen}

En este artículo se propone la linealización de un sistema Radio-over-Fiber (RoF) de doble banda mediante predistorsión digital. Los resultados han sido evaluados experimentalmente con señales LTE en un sistema RoF, obteniendo mejores resultados que con el predistorsionador clásico de una banda en términos de Adjacent Channel Power Ratio (ACPR), pérdidas de potencia y Error Vector Magnitude (EVM).

\section{Introducción}

En la actualidad, las conexiones entre la Baseband Unit (BBU) y las diferentes Remote Radio Heads (RRH) en la arquitectura Cloud Radio Access Networks (C-RAN) son llevadas a cabo mediante enlaces Radio-over-Fiber (RoF) [1]. Los enlaces de RoF son transmisiones analógicas, y por lo tanto susceptibles de no linealidades [2]. A lo largo de la literatura se han propuesto tanto métodos fotónicos como eléctricos para corregir las distorsiones, siendo la predistorsión digital (DPD) una de las más efectivas cancelando los productos de intermodulación.

Sin embargo, el comportamiento no lineal del transmisor es mucho más notable en sistemas de doble banda, en los cuales dos señales son transmitidas simultáneamente. En este trabajo se presenta un predistorsionador bibanda (2D-DPD), en el cual se tienen en cuenta tanto las distorsiones de la propia banda como las de modulación cruzada. Los experimentos son llevados a cabo en un sistema RoF multibanda con señales del estándar LTE.

\section{Modelos DPD propuestos}

El predistorsionador de una sola banda (1D-DPD) utilizado en este trabajo está basado en los modelos polinomiales clásicos. Una descripción más detallada de este modelo puede encontrarse en [3]. En el modelo 2D-DPD se tienen en cuenta tanto los productos de intermodulación dentro de la banda como los de modulación cruzada. Por lo tanto, se tienen en cuenta ambas señales moduladas en diferentes frecuencias a la hora de identificar los coeficientes del predistorsionador (ver Fig. 1) [4]. Este modelo está definido como

$$
\begin{aligned}
& u_{1}(n)=\sum_{m=0}^{M-1} \sum_{k=0}^{N-1} \sum_{l=0}^{k} c_{m, k, l}^{(1)} x_{1}(n-m) \times\left|x_{1}(n-m)\right|^{k-l}\left|x_{2}(n-m)\right|^{l} \\
& u_{2}(n)=\sum_{m=0}^{M-1} \sum_{k=0}^{N-1} \sum_{l=0}^{k} c_{m, k, l}^{(2)} x_{2}(n-m) \times\left|x_{2}(n-m)\right|^{k-l}\left|x_{1}(n-m)\right|^{l}
\end{aligned}
$$

donde $M$ es la profundidad de memoria, $N$ el orden de no linealidad, $x_{1}(n-m)$ y $x_{2}(n-m)$ son las señales de entrada al DPD en banda base a las frecuencias de portadora $\omega_{1}$ y $\omega_{2}$, respectivamente. $u_{1}(n)$ y $u_{2}(n)$ son las señales de salida del DPD en banda base y $c_{m, k, l}^{(1)}$ y $c_{m, k, l}^{(2)}$ son los vectores de coeficientes del modelo propuesto.

\section{Resultados experimentales}

El modelo 2D-DPD es evaluado en un sistema RoF. En el lado de la RRH hay dos generadores de señal sincronizados en fase $y$ en tiempo, cuyas frecuencias de portadora son 798 y $867.5 \mathrm{MHz}$, respectivamente. Las señales de salida de los generadores son combinadas, para después alimentar un electro-absorption modulator (EAM) distributed feedback laser (DFB). La transmisión óptica entre la BBU y la RRH consiste en $10 \mathrm{~km}$ de fibra monomodo. En el lado de la RRH, la conversión óptico-eléctrica es llevada a cabo por un fotodetector, cuya salida es amplificada con un amplificador de potencia (PA). Las dos señales de salida del sistema RoF son capturadas por separado con un osciloscopio digital, y después son alineadas con sus correspondientes señales de entrada. Finalmente se calculan los coeficientes del modelo en un PC con un orden de no linealidad de $\mathrm{N}=3$ y una profundidad de memoria de $\mathrm{M}=1$. Estas formas de onda consisten en dos señales LTE downlink con subportadoras QPSK y 16QAM cuyos anchos de banda son 10 y $15 \mathrm{MHz}$, respectivamente. 
Tabla 1. Resultados experimentales evaluados con ambos predistorsionadores (1D-DPD y 2D-DPD)

\begin{tabular}{|c|c|c|c|c|c|c|}
\hline \multirow{2}{*}{ Case } & \multicolumn{2}{|c|}{ ACPR $(\mathrm{dBc})$} & \multicolumn{2}{|c|}{ Losses $(\mathrm{dB})$} & \multicolumn{2}{|c|}{ EVM (\%) } \\
\hline & Band 1 & Band 2 & Band 1 & Band 2 & Band 1 & Band 2 \\
\hline w/o DPD & -29.58 & -27.41 & - & - & 8.74 & 7.64 \\
\hline 1D-DPD & -29.51 & -28.54 & 2.87 & 2.64 & 6.93 & 5.09 \\
\hline 2D-DPD & -33.20 & -32.86 & 2.55 & 1.93 & 4.64 & 3.21 \\
\hline
\end{tabular}

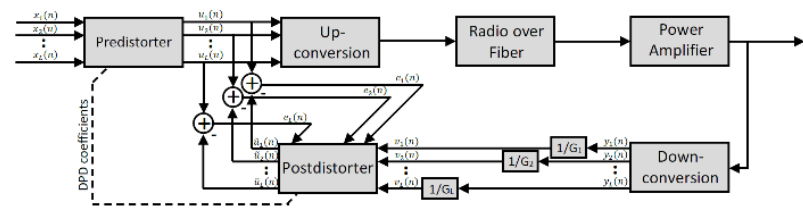

Figura 2. Esquema del DPD multi-banda en un sistema RoF

Los resultados experimentales están resumidos en la Tabla 1, donde el DPD de una banda sólo es capaz de corregir las no linealidades en parte en términos de ACPR, mientras que con el predistorsionador de doble banda es capaz de reducir el recrecimiento espectral en $4 \mathrm{~dB}$ respecto del clásico de una banda. Además, las pérdidas introducidas por el predistorsionador son menores con el 2D-DPD. En términos de EVM, con el predistorsionador propuesto su valor se reduce de $8.74 \%$ y $7.64 \%$ a $4.64 \%$ y $3.21 \%$ en las dos bandas, respectivamente, cumpliendo los límites marcados por el estándar LTE [5]. En la Fig. 2 están las densidades espectrales de potencia y las constelaciones de las señales de salida del sistema RoF para las dos bandas.

\section{Conclusiones}

En este artículo se ha evaluado experimentalmente un predistorsionador doble banda en un sistema RoF con bandas concurrentes. Según los resultados experimentales, el DPD de una banda solamente puede corregir las distorsiones de una banda, mientras que con el 2D-DPD propuesto se corrigen tanto las distorsiones dentro de la banda como las de las bandas cruzadas. Los resultados son evaluados en términos de ACPR, pérdidas de potencia y EVM.

\section{REFERENCIAS}

[1]. PFEIFFER, T. Next generation Mobile Fronthaul Architectures, Proceedings of the Optical Fiber Communications Conference. Los Ángeles, 2015, paper M2J.7.
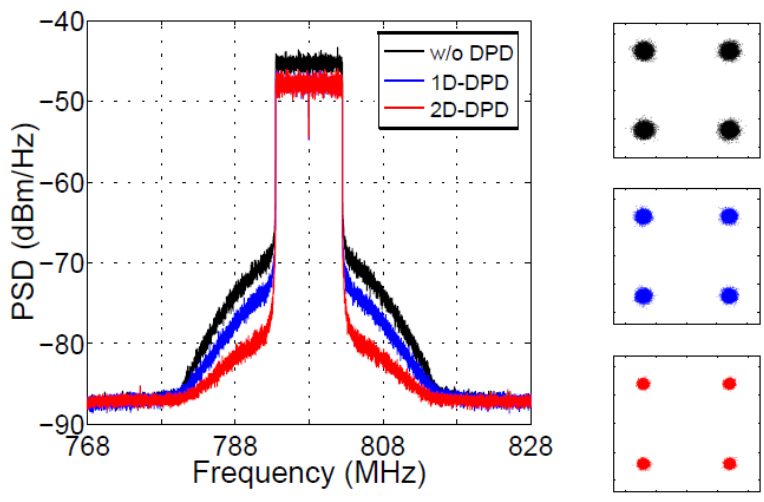

(a)
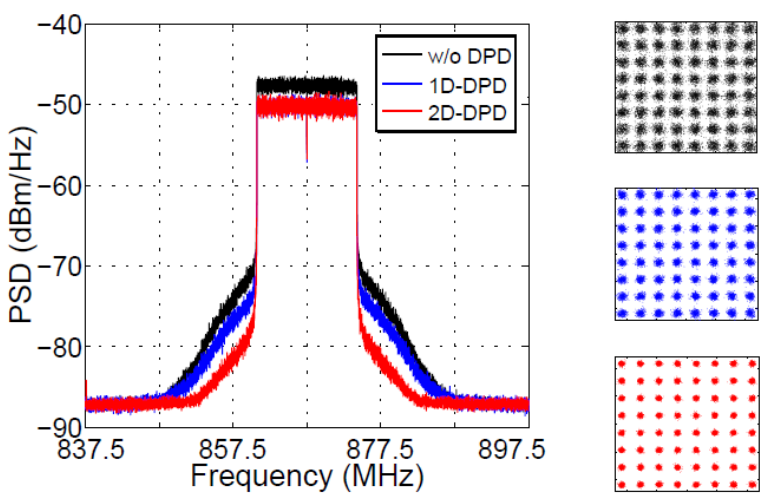

(b)

Figura 2. PSD de la Banda 1 (a) y Banda 2 (b) sin DPD (negro), con 1D-DPD (azul) y con 2D-DPD (rojo).

[2]. ALIC, N. Cancellation of Nonlinear Impairments in Fiber Optic Transmission Systems. Proceedings of the Optical Fiber Communications Conference. Anaheim, 2016, paper Tu2E.1.

[3]. ZHANG, J. et al. Memory-Polynomial Digital Predistortion for Linearity Improvement of DirectlyModulated Multi-IF-over-Fiber LTE Mobile Fronthaul. Proceedings of the Optical Fiber Communications Conference. Anaheim, 2016, paper Tu2B.3.

[4]. BASSAM, S. A. et al. 2-D Digital Predistortion (2-DDPD) Architecture for Concurrent Dual-Band Transmitters. IEEE Trans. Microw. Theory Techn. 2011, 59(10), 2547-2553.

[5]. 3GPP, Base Station (BS) transmission and reception, Technical Specification ETSI TS136.104 V13.3 